\title{
DRG \& Selbsthilfegruppen zum Thema „Gadolinium-haltige Kontrastmittel“
}

In den vergangenen Jahren wurde verstärkt über die bei der Gabe von Gadolinium-haltigen Kontrastmitteln beobachteten Gewebeablagerungen berichtet und gleichzeitig bei Patientinnen und Patienten auftretende Nebenwirkungen damit in Zusammenhang gebracht.

In einem Gespräch mit Vertreterinnen und Vertretern der Deutschen Röntgengesellschaft e. V. (DRG) berichteten am 3. März 2020 Patientinnen und Patienten aus Selbsthilfeorganisationen über gravierende Symptome aus dem neurologischen, dermatologischen und inflammatorischen Formenkreis, die im zeitlichen Zusammenhang mit der Gabe von Gadolinium-haltigen Kontrastmitteln aufgetreten sind.

Die Selbsthilfeorganisationen und die DRG fordern ihre Mitglieder auf, für die Meldung der beobachteten Nebenwirkungen die dafür vom Bundesinstitut für Arzneimittel und Medizinprodukte (BfArM) online zur Verfügung gestellten Formulare zu nutzen (Ärzte: www.humanweb.pei.de; Verbraucher: www.nebenwirkungen.pei.de). Nur so ist ein Überblick über die Art und das Ausmaß der beobachteten Nebenwirkungen möglich. Beide Seiten vereinbarten die Fortsetzung der Diskussion.

An dem Gespräch nahmen teil (in alphabetischer Reihenfolge): Kurt A., Prof. Dr. Gerald Antoch, Antje B., Daniel H., Claudia K., Bruno L., Dr. Stefan Lohwasser, Cornelia M., Prof. Dr. Henrik Michaely, Maren O., Prof. Dr. Alexander Radbruch, Prof. Dr. Diane Renz, Heiko T., Sabine T., Prof. Dr. Michael Uder, Angelika U. 